UDC 517.9

\title{
CLASSIFICATION AND EXISTENCE OF NONOSCILLATORY SOLUTIONS OF SECOND-ORDER NEUTRAL DELAY DYNAMIC EQUATIONS ON TIME SCALES
}

\section{КЛАСИФІКАЦІЯ ТА ІСНУВАННЯ НЕКОЛИВНИХ РОЗВ'ЯЗКІВ ДИНАМІЧНИХ РІВНЯНЬ ДРУГОГО ПОРЯДКУ З НЕЙТРАЛЬНИМ ЗАПІЗНЕННЯМ НА ЧАСОВІЙ ШКАЛІ}

\author{
Yong Zhou, Yonghong Lan \\ Xiangtan Univ. \\ Hunan 411105, P. R. China \\ e-mail:yzhou@xtu.edu.cn
}

In this paper, we give a classification of nonoscillatory solutions of the second-order neutral delay dynamic equation on time scales

$$
[x(t)-c(t) x(t-\tau)]^{\Delta \Delta}+f\left(t, x\left(g_{1}(t)\right), \ldots, x\left(g_{m}(t)\right)\right)=0, \quad t \in T .
$$

Some existence results for each kind of nonoscillatory solutions are also established.

Наведено класифікацію неколивних розв'язків динамічних рівнянь другого порядку з нейтральним запізненням на часовій шкалі

$$
[x(t)-c(t) x(t-\tau)]^{\Delta \Delta}+f\left(t, x\left(g_{1}(t)\right), \ldots, x\left(g_{m}(t)\right)\right)=0, \quad t \in T,
$$

а також доведено існування неколивних розв'язків кожного типу.

1. Introduction. The theory of time scales was introduced by Hilger [10] in 1988 in order to unify continuous and discrete analysis. Recently, the study of dynamic equations on time scales has received a lot of attention. The general idea is to prove a result for a dynamic equation where the domain of the unknown function is a so-called time scale, which is a special case of a measure chain. By choosing the time scale to be the set of real numbers, the general results yields a result concerning a differential equation. On the other hand, by choosing the time scale to be the set of integers, the same general result yields a result for difference equations. However, since there are many other time scales than just the set of real numbers or the set of integers, one has a much more general results. The monographs by Bohner and Peterson $[4,5]$ and the survey on dynamic equations on time scales by Agarwal, Bohner, O'Regan and Peterson [2] summarized some important work in this area. Recently, the oscillation of dynamic equations on time scales has received much attention (see $[6-8,12-18]$ ). But the classification and existence of nonoscillatory solutions of the delay dynamic equations on time scales received much less attention.

* Project supported by National Natural Science Foundation of P.R. China (11271309), the Specialized Research Fund for the Doctoral Program of Higher Education (20114301110001) and Hunan Provincial Natural Science Foundation of China (12JJ2001).

(C) Yong Zhou, Yonghong Lan, 2013

ISSN 1562-3076. Нелінійні коливання, 2013, m. 16, N으 
In this paper, we consider the second-order neutral delay dynamic equation on time scales

$$
[x(t)-c(t) x(t-\tau)]^{\Delta \Delta}+f\left(t, x\left(g_{1}(t)\right), \ldots, x\left(g_{m}(t)\right)\right)=0,
$$

where $t \in\left[t_{0}, \infty\right)=T_{0} \subseteq T$. With respect to (1.1), throughout we shall assume the following:

$\left(\mathrm{H}_{1}\right) \tau>0, c(t) \in C_{r d}\left(T_{0}, \mathbf{R}_{+}\right), \mathbf{R}_{+}=[0, \infty)$, there exists $\delta \in(0,1]$ such that $c(t) \leq 1-\delta$ for $t \in T_{0}$.

$\left(\mathrm{H}_{2}\right) g_{i} \in C_{r d}\left(T_{0}, \mathbf{R}_{+}\right)$, and $\lim _{t \rightarrow \infty} g_{i}(t)=\infty, i=1,2, \ldots, m$.

$\left(\mathrm{H}_{3}\right) f: T_{0} \times \mathbf{R}^{m} \rightarrow \mathbf{R}$ is right-dense continuous on $T_{0}$ and continuous with respect to the last $m$ arguments, $y_{1} f\left(t, y_{1}, \ldots, y_{m}\right)>0$ for $y_{1} y_{i}>0, i=2, \ldots, m$. Moreover,

$$
\left|f\left(t, x_{1}, \ldots, x_{m}\right)\right| \geq\left|f\left(t, y_{1}, \ldots, y_{m}\right)\right|
$$

when $\left|y_{i}\right| \leq\left|x_{i}\right|$ and $x_{i} y_{i}>0, i=1,2, \ldots, m$.

For convenience, we set

$$
y(t)=x(t)-c(t) x(t-\tau) .
$$

In Section 3, we will study the existence and asymptotic behavior of nonoscillatory solutions of equation (1.1). More precisely, we give a classification of nonoscillatory solutions of equation (1.1) according to their asymptotic behavior. Moreover, we established some existence results for each kind of nonoscillatory solutions of equation (1.1). In particular, we obtain two necessary and sufficient conditions for the existence of nonoscillatory solutions of (1.1).

2. Preliminaries. To understand the delay dynamic equations on time scales we need some preliminary definitions (see [4]).

Let $T$ be a time scale (i.e., a closed subset of the real numbers $\mathbf{R}$ ) with $\sup T=\infty$. We assume throughout that $T$ has the topology that it inherits from the standard topology on the real numbers $\mathbf{R}$.

Definition 2.1. For $t \in T$ we define the forward jump operator $\sigma: T \rightarrow T$ by

$$
\sigma(t):=\inf \{s \in T: s>t\}
$$

while the backwards jump operator $\rho: T \rightarrow T$ is defined by

$$
\rho(t):=\sup \{s \in T: s<t\} .
$$

If $\sigma(t)>t$, we say that $t$ is right-scattered, while if $\rho(t)<t$ we say that $t$ is left-scattered. Points that are right-scattered and left-scattered at the same time are called isolated. Also, if $t<\sup T$ and $\sigma(t)=t$, then $t$ is called right-dense, and if $t>\inf T$ and $\rho(t)=t$, then $t$ is called left-dense. Points that are right-dense and left-dense at same time are called dense.

Definition 2.2. Define the interval in $T$

$$
[a, b]:=\{t \in T \quad \text { such that } a \leq t \leq b\}
$$

Open intervals and half-open intervals etc. are defined accordingly. Note that $[a, b]^{K}=[a, b]$ if $b$ is left-dense and $[a, b]^{K}=[a, b)=[a, \rho(b)]$ if $b$ is left-scattered. 
Definition 2.3. Assume $f: T \rightarrow \mathbf{R}$ and let $t \in T$ (ift $=\sup T$ assume $t$ is not left-scattered), then we define $f^{\Delta}(t)$ to be the number (provided it exists) with the property that given any $\epsilon>0$, there is a neighborhood $U$ of $t$ (i.e., $U=(t-\delta, t+\delta) \cap T$ for some $\delta>0)$ such that

$$
\left|[f(\sigma(t))-f(s)]-f^{\Delta}(t)[\sigma(t)-s]\right| \leq \epsilon|\sigma(t)-s|, \quad \text { for all } \quad s \in U .
$$

We call $f^{\Delta}(t)$ the delta (or Hilger) derivative of $f$ at $t$.

It can be shown that if $f: T \rightarrow \mathbf{R}$ is continuous at $t \in T$ and $t$ is right-scattered, then

$$
f^{\Delta}(t)=\frac{f(\sigma(t))-f(t)}{\mu(t)}
$$

If $t$ is right-dense, then

$$
f^{\Delta}(t)=\lim _{s \rightarrow t} \frac{f(\sigma(t))-f(s)}{t-s} .
$$

Lemma 2.1. Assume $g: T \rightarrow R$ to be differentiable and $g^{\Delta}(t) \geq 0$. Then $g(t)$ is nondecreasing.

Definition 2.4. We say $f: T \rightarrow \mathbf{R}$ is right-dense continuous on $T$ provided it is continuous at all right-dense points and at points that are left-dense and right-scattered we just assume the left hand limit exists (and is finite). We denote this by $f \in C_{r d}(T, \mathbf{R})$.

Lemma 2.2. Assume $f: T \rightarrow R$ to be differentiable at $t$, then $f$ is continuous at $t$.

Definition 2.5. If $F^{\Delta}(t)=f(t)$, then we define an integral by

$$
\int_{a}^{t} f(\tau) \Delta \tau:=F(t)-F(a) .
$$

Definition 2.6. If $a \in T$ and $f \in C_{r d}([a, \infty), \mathbf{R})$, then we define the improper integral by

$$
\int_{a}^{\infty} f(t) \Delta t:=\lim _{b \rightarrow \infty} \int_{a}^{b} f(t) \Delta t
$$

provided this limit exists, and we say that the improper integral converges in this case. If this limit does not exist, then we say that the improper integral diverges.

Lemma 2.3. Let $a \in T^{K}, b \in T$ and assume $f: T \times T^{K} \rightarrow \mathbf{R}$ is continuous at $(t, t)$, where $t \in T^{K}$ with $t>a$. Also assume that $f^{\Delta}(t, \cdot)$ is rd-continuous on $[a, \sigma(t)]$. Suppose that for each $\varepsilon>0$ there exists a neighborhood $U$ of $t$, independent of $\tau \in[a, \sigma(t)]$, such that

$$
\left|f(\sigma(t), \tau)-f(s, \tau)-f^{\Delta}(t, \tau)(\sigma(t)-s)\right| \leq \varepsilon|\sigma(t)-s|, \quad \text { for all } \quad s \in U,
$$

where $f^{\Delta}$ denotes the derivative of $f$ with respect to the first variable. Then

(i) $g(t):=\int_{a}^{t} f(t, \tau) \Delta \tau$ implies $g^{\Delta}(t)=\int_{a}^{t} f^{\Delta}(t, \tau) \Delta \tau+f(\sigma(t), t)$; 
(ii) $h(t):=\int_{t}^{b} f(t, \tau) \Delta \tau$ implies $h^{\Delta}(t)=\int_{t}^{b} f^{\Delta}(t, \tau) \Delta \tau-f(\sigma(t), t)$.

Definition 2.7. A solution of (1.1) is said to be oscillatory if it is neither eventually positive nor eventually negative, otherwise it is nonoscillatory.

3. Main results. First we show some lemmas which will be useful for the main results of this section.

Lemma 3.1. Let $x(t)$ be an eventually positive (or negative) solution of (1.1). If $\lim _{t \rightarrow \infty} x(t)=$ $=0$, then $y(t)$ is eventually negative (or positive) and $\lim _{t \rightarrow \infty} y(t)=0$. If $\lim _{t \rightarrow \infty} x(t)=0$ fails, then $y(t)$ is eventually positive (or negative).

Proof. Let $x(t)$ be an eventually positive solution of (1.1). From (1.1), $y^{\Delta \Delta}(t)<0$ eventually. Thus $y^{\Delta}(t)$ is decreasing and $y^{\Delta}(t)>0$ or $y^{\Delta}(t)<0$ eventually. Also, $y(t)>0$ or $y(t)<0$ eventually. If $\lim _{t \rightarrow \infty} x(t)=0$, from (1.2) we have $\lim _{t \rightarrow \infty} y(t)=0$. Since $y(t)$ is monotonic, so $\lim _{t \rightarrow \infty} y^{\Delta}(t)=0$, which implies that $y^{\Delta}(t)>0$. Therefore, $y(t)<0$ eventually. If $\lim _{t \rightarrow \infty} x(t)=0$ fail, then $\limsup _{t \rightarrow \infty} x(t)>0$. We show that $y(t)>0$ eventually. If not, then $y(t)<0$ eventually. If $x(t)$ is unbounded, then there exists a sequence $\left\{t_{n}\right\}$ such that $\lim _{n \rightarrow \infty} t_{n}=\infty, x\left(t_{n}\right)=\max _{t_{0} \leq t \leq t_{n}} x(t)$ and $\lim _{n \rightarrow \infty} x\left(t_{n}\right)=\infty$. From (1.2), we have

$$
y\left(t_{n}\right)=x\left(t_{n}\right)-c\left(t_{n}\right) x\left(t_{n}-\tau\right) \geq x\left(t_{n}\right)\left(1-c\left(t_{n}\right)\right) .
$$

Thus $\lim _{n \rightarrow \infty} y\left(t_{n}\right)=\infty$, which is a contradiction. If $x(t)$ is bounded, then there exists a sequence $\left\{t_{n}\right\}$ such that $\lim _{n \rightarrow \infty} t_{n}=\infty$ and $\lim _{n \rightarrow \infty} x\left(t_{n}\right)=\limsup _{t \rightarrow \infty} x(t)$. Since the sequences $\left\{c\left(t_{n}\right)\right\}$ and $\left\{x\left(t_{n}-\tau\right)\right\}$ are bounded, there exist convergent subsequences. Without loss of generality, we may assume that $\lim _{n \rightarrow \infty} x\left(t_{n}-\tau\right)$ and $\lim _{n \rightarrow \infty} c\left(t_{n}\right)$ exist. Hence

$$
0 \geq \lim _{n \rightarrow \infty} y\left(t_{n}\right)=\lim _{n \rightarrow \infty}\left(x\left(t_{n}\right)-c\left(t_{n}\right) x\left(t_{n}-\tau\right)\right) \geq \limsup _{t \rightarrow \infty} x(t)\left(1-\lim _{n \rightarrow \infty} c\left(t_{n}\right)\right)>0
$$

which is a contradiction again. Therefore, $y(t)>0$ eventually. A similar proof can be given if $x(t)<0$ eventually.

Lemma 3.2. Assume that $\lim _{t \rightarrow \infty} c(t)=c \in[0,1)$, and $x(t)$ is an eventually positive (or negative) solution of (1.1). If $\lim _{t \rightarrow \infty} y(t)=a \in R$, then $\lim _{t \rightarrow \infty} x(t)=\frac{a}{1-c}$. If $\lim _{t \rightarrow \infty} y(t)=\infty$ $($ or $-\infty)$, then $\lim _{t \rightarrow \infty} x(t)=\infty($ or $-\infty)$.

Proof. Let $x(t)$ be an eventually positive solution of (1.1). Then $x(t) \geq y(t)$ eventually. If $\lim _{t \rightarrow \infty} y(t)=\infty$, then $\lim _{t \rightarrow \infty} x(t)=\infty$. Now we consider the case that $\lim _{t \rightarrow \infty} y(t)=a \in$ $\in R$. Thus $y(t)$ is bounded which implies that $x(t)$ is bounded (see (3.1)). Therefore, there exists a sequence $\left\{t_{n}\right\}$ such that $\lim _{n \rightarrow \infty} t_{n}=\infty$ and $\lim _{n \rightarrow \infty} x\left(t_{n}\right)=\limsup _{t \rightarrow \infty} x(t)$. As before, without loss of generality, we may assume that $\lim _{n \rightarrow \infty} c\left(t_{n}\right)$ and $\lim _{n \rightarrow \infty} x\left(t_{n}-\tau\right)$ exist. Hence

$$
a=\lim _{n \rightarrow \infty} y\left(t_{n}\right)=\lim _{n \rightarrow \infty} x\left(t_{n}\right)-\lim _{n \rightarrow \infty} c\left(t_{n}\right) \lim _{n \rightarrow \infty} x\left(t_{n}-\tau\right) \geq \limsup _{t \rightarrow \infty} x(t)(1-c)
$$

i.e.,

$$
\frac{a}{1-c} \geq \limsup _{t \rightarrow \infty} x(t)
$$


On the other hand, there exists $\left\{t_{n}^{\prime}\right\}$ such that $\lim _{n \rightarrow \infty} x\left(t_{n}^{\prime}\right)=\liminf _{t \rightarrow \infty} x(t)$. Without loss of generality, we assume that $\lim _{n \rightarrow \infty} c\left(t_{n}^{\prime}\right)$ and $\lim _{n \rightarrow \infty} x\left(t_{n}^{\prime}-\tau\right)$ exist. Hence

$$
a=\lim _{n \rightarrow \infty} y\left(t_{n}^{\prime}\right)=\lim _{n \rightarrow \infty} x\left(t_{n}^{\prime}\right)-\lim _{n \rightarrow \infty} c\left(t_{n}^{\prime}\right) \lim _{n \rightarrow \infty} x\left(t_{n}^{\prime}-\tau\right) \leq \liminf _{t \rightarrow \infty} x(t)(1-c)
$$

or

$$
\frac{a}{1-c} \leq \liminf _{t \rightarrow \infty} x(t)
$$

Combining (3.2) and (3.3) we obtain $\lim _{t \rightarrow \infty} x(t)=\frac{a}{1-c}$. A similar proof can be given if $x(t)<0$.

We are now ready to prove the following results.

Theorem 3.1. Assume that $\lim _{t \rightarrow \infty} c(t)=c \in[0,1)$. Let $x(t)$ be a nonoscillatory solution of (1.1). Let $E$ denote the set of all nonoscillatory solution of (1.1), and define

$$
\begin{aligned}
& E(0,0,0)=\left\{x(t) \in E: \lim _{t \rightarrow \infty} x(t)=0, \lim _{t \rightarrow \infty} y(t)=0, \lim _{t \rightarrow \infty} y^{\Delta}(t)=0\right\}, \\
& E(b, a, 0)=\left\{x(t) \in E: \lim _{t \rightarrow \infty} x(t)=b=\frac{a}{1-c}, \lim _{t \rightarrow \infty} y(t)=a, \lim _{t \rightarrow \infty} y^{\Delta}(t)=0\right\}, \\
& E(\infty, \infty, 0)=\left\{x(t) \in E: \lim _{t \rightarrow \infty} x(t)=\infty, \lim _{t \rightarrow \infty} y(t)=\infty, \lim _{t \rightarrow \infty} y^{\Delta}(t)=0\right\}, \\
& E(\infty, \infty, d)=\left\{x(t) \in E: \lim _{t \rightarrow \infty} x(t)=\infty, \lim _{t \rightarrow \infty} y(t)=\infty, \lim _{t \rightarrow \infty} y^{\Delta}(t)=d \neq 0\right\} .
\end{aligned}
$$

Then

$$
E=E(0,0,0) \cup E(b, a, 0) \cup E(\infty, \infty, 0) \cup E(\infty, \infty, d) .
$$

Proof. Without loss of generality, let $x(t)$ be an eventually positive solution of (1.1). If $\lim _{t \rightarrow \infty} x(t)=0$, by Lemma 3.1, $\lim _{t \rightarrow \infty} y(t)=0$ and $\lim _{t \rightarrow \infty} y^{\Delta}(t)=0$, i.e., $x(t) \in E(0,0,0)$. If $\lim _{t \rightarrow \infty} x(t)=0$ fails, then by Lemma 3.1,y(t)>0 eventually, and it is easy to see that $y^{\Delta}(t)>0, y^{\Delta \Delta}(t)<0$ eventually. If $\lim _{t \rightarrow \infty} y(t)=a>0$ exists, then $\lim _{t \rightarrow \infty} y^{\Delta}(t)=0$, by Lemma 3.2, and we have $\lim _{t \rightarrow \infty} x(t)=\frac{a}{1-c}=b$, i.e., $x(t) \in E(b, a, 0)$. If $\lim _{t \rightarrow \infty} y(t)=\infty$, then by Lemma $3.2 \lim _{t \rightarrow \infty} x(t)=\infty$. Since $y^{\Delta \Delta}(t)<0$ and $y^{\Delta}(t)>0$, we have $\lim _{t \rightarrow \infty} y^{\Delta}(t)=d$, where $d=0$ or $d>0$. Then either $x(t) \in E(\infty, \infty, 0)$, or $x(t) \in$ $\in E(\infty, \infty, d)$.

In the following we shall show some existence results for each kind of nonoscillatory solution of Eq. (1.1).

Theorem 3.2. Assume that $\lim _{t \rightarrow \infty} c(t)=c \in[0,1)$. Then Eq.(1.1) has a nonoscillatory solution $x(t) \in E(b, a, 0)(b \neq 0, a \neq 0)$ if and only if

$$
\int_{t_{0}}^{\infty} \sigma(u)\left|f\left(u, b_{1}, \ldots, b_{1}\right)\right| \Delta u<\infty \quad \text { for some } \quad b_{1} \neq 0 .
$$


Proof. Necessity. Without loss of generality, let $x(t) \in E(b, a, 0)$ be an eventually positive solution of (1.1). By Theorem 3.1 we know that $b>0, a>0$. From (1.1) and (1.2) we have

$$
y^{\Delta \Delta}(t)=-f\left(t, x\left(g_{1}(t)\right), \ldots, x\left(g_{m}(t)\right)\right) .
$$

Integrating it from $s$ to $\infty$ for $s \geq t_{0}$ we obtain

$$
y^{\Delta}(s)=\int_{s}^{\infty} f\left(u, x\left(g_{1}(u)\right), \ldots, x\left(g_{m}(u)\right)\right) \Delta u .
$$

Integrating it from $t_{1}$ to $t$ for $t_{1}$ sufficiently large, we get

$$
\begin{aligned}
y(t)= & y\left(t_{1}\right)+\int_{t_{1}}^{t}\left(\sigma(u)-t_{1}\right) f\left(u, x\left(g_{1}(u)\right), \ldots, x\left(g_{m}(u)\right)\right) \Delta u+ \\
& +\int_{t}^{\infty}\left(t-t_{1}\right) f\left(u, x\left(g_{1}(u)\right), \ldots, x\left(g_{m}(u)\right)\right) \Delta u .
\end{aligned}
$$

Since $\lim _{u \rightarrow \infty} x\left(g_{i}(u)\right)=b>0, i=1,2, \ldots, m$, there exists an $t_{1} \geq t_{0}$ such that $x\left(g_{i}(u)\right) \geq \frac{b}{2}$ for $t \geq t_{1}$. Hence we have

$$
\int_{t_{1}}^{t}\left(\sigma(u)-t_{1}\right)\left|f\left(u, \frac{b}{2}, \ldots, \frac{b}{2}\right)\right| \Delta u<y(t)-y\left(t_{1}\right)
$$

which implies that (3.4) holds.

Sufficiency. Set $b_{1}>0$ and $A>0$ so that $A<(1-c) b_{1}$. From (3.4) there exists a sufficiently large $t_{1}$ so that for $t \geq t_{1}$ we have $t-\tau \geq t_{0}$ and $g_{i}(t) \geq t_{0}, i=1,2, \ldots, m$, and

$$
\frac{A}{b_{1}}+c(t)+\frac{1}{b_{1}} \int_{t_{1}}^{\infty} \sigma(u) f\left(u, b_{1}, \ldots, b_{1}\right) \Delta u \leq 1 .
$$

Let $X$ denote the Banach space of all bounded rd-continuous functions $x(t)$ on $\left[t_{0}, \infty\right)$ with the norm $\|x(t)\|=\sup _{t \geq t_{0}}|x(t)|<\infty$. Define a set $\Omega$ by

$$
\Omega=\left\{x(t) \in X \mid 0 \leq x(t) \leq b_{1}, t \geq t_{0}\right\}
$$

and an operator $S$ on $\Omega$ by

$$
(S x)(t)=\left\{\begin{array}{l}
A+c(t) x(t-\tau) \\
+\int_{t_{1}}^{t} \sigma(u) f\left(u, x\left(g_{1}(u)\right), \ldots, x\left(g_{m}(u)\right)\right) \Delta u+ \\
+\int_{t}^{\infty} t f\left(u, x\left(g_{1}(u)\right), \ldots, x\left(g_{m}(u)\right)\right) \Delta u, \quad \text { if } t \geq t_{1}, \\
(S x)\left(t_{1}\right), \quad \text { if } \quad t_{0} \leq t<t_{1} .
\end{array}\right.
$$


CLASSIFICATION AND EXISTENCE OF NONOSCILLATORY SOLUTIONS OF SECOND-ORDER NEUTRAL DELAY ... 197

Clearly, for $x(t) \in \Omega$,

$$
\begin{aligned}
(S x)(t) & \leq A+c(t) b_{1}+\int_{t_{1}}^{t} \sigma(u) f\left(u, b_{1}, \ldots, b_{1}\right) \Delta u+\int_{t}^{\infty} \sigma(u) f\left(u, b_{1}, \ldots, b_{1}\right) \Delta u \leq \\
& \leq A+c(t) b_{1}+\int_{t_{1}}^{\infty} \sigma(u) f\left(u, b_{1}, \ldots, b_{1}\right) \Delta u \leq b_{1}, \quad t \geq t_{1},
\end{aligned}
$$

and

$$
(S x)(t)=(S x)\left(t_{1}\right) \leq b_{1}, \quad t_{0} \leq t \leq t_{1},
$$

i.e., $S \Omega \subset \Omega$.

Define a series of sequences $\left\{x_{k}(t)\right\}, k \in \mathbf{N}_{0}$, as

$$
\begin{gathered}
x_{0}(t)=0 \\
x_{k}(t)=\left(S x_{k-1}\right)(t), \quad k \in \mathbf{N}, \quad t \geq t_{0} .
\end{gathered}
$$

By induction, we can prove that

$$
0 \leq x_{k}(t) \leq x_{k+1}(t) \leq b_{1}, \quad t \geq t_{0}, \quad k \in \mathbf{N}_{\mathbf{0}} .
$$

Then there exists $x(t) \subset \Omega$ such that $\lim _{t \rightarrow \infty} x_{k}(t)=x(t), t \geq t_{0}$.

In the following, we shall show that

$$
\lim _{k \rightarrow \infty} \int_{t}^{\infty} t f\left(u, x_{k}\left(g_{1}(u)\right), \ldots, x_{k}\left(g_{m}(u)\right)\right) \Delta u=\int_{t}^{\infty} t f\left(u, x\left(g_{1}(u)\right), \ldots, x\left(g_{m}(u)\right)\right) \Delta u .
$$

In fact, by (3.4), for any $\varepsilon>0$ there exists $t_{1} \geq t_{0}$ such that

$$
\int_{t_{1}}^{\infty} \sigma(u) f\left(u, b_{1}, \ldots, b_{1}\right) \Delta u<\varepsilon
$$

Thus, for $t_{2} \geq t_{1}$ we get

$$
\begin{aligned}
& \left|\int_{t}^{t_{2}} t f\left(u, x_{k}\left(g_{1}(u)\right), \ldots, x_{k}\left(g_{m}(u)\right)\right) \Delta u-\int_{t}^{\infty} t f\left(u, x_{k}\left(g_{1}(u)\right), \ldots, x_{k}\left(g_{m}(u)\right)\right) \Delta u\right|= \\
& \quad=\left|\int_{t_{2}}^{\infty} t f\left(u, x_{k}\left(g_{1}(u)\right), \ldots, x_{k}\left(g_{m}(u)\right)\right) \Delta u\right| \leq \int_{t_{2}}^{\infty} \sigma(u) f\left(u, x_{k}\left(g_{1}(u)\right), \ldots, x_{k}\left(g_{m}(u)\right)\right) \Delta u \leq \\
& \quad \leq \int_{t_{2}}^{\infty} \sigma(u) f\left(u, b_{1}, \ldots, b_{1}\right) \Delta u<\varepsilon .
\end{aligned}
$$


Hence, $\int_{t}^{t_{2}} t f\left(u, x_{k}\left(g_{1}(u)\right), \ldots, x_{k}\left(g_{m}(u)\right)\right) \Delta u \rightarrow \int_{t}^{\infty} t f\left(u, x_{k}\left(g_{1}(u)\right), \ldots, x_{k}\left(g_{m}(u)\right)\right) \Delta u$ uniformly for $k \in N$ as $t_{2} \rightarrow \infty$. Therefore,

$$
\begin{aligned}
\lim _{k \rightarrow \infty} \int_{t}^{\infty} t f\left(u, x_{k}\left(g_{1}(u)\right)\right. & \left.\ldots, x_{k}\left(g_{m}(u)\right)\right) \Delta u= \\
& =\lim _{k \rightarrow \infty} \lim _{t_{2} \rightarrow \infty} \int_{t}^{t_{2}} t f\left(u, x_{k}\left(g_{1}(u)\right), \ldots, x_{k}\left(g_{m}(u)\right)\right) \Delta u= \\
& =\lim _{t_{2} \rightarrow \infty} \lim _{k \rightarrow \infty} \int_{t}^{t_{2}} t f\left(u, x_{k}\left(g_{1}(u)\right), \ldots, x_{k}\left(g_{m}(u)\right)\right) \Delta u= \\
& =\lim _{t_{2} \rightarrow \infty} \int_{t}^{t_{2}} t f\left(u, x\left(g_{1}(u)\right), \ldots, x\left(g_{m}(u)\right)\right) \Delta u= \\
& =\int_{t}^{\infty} t f\left(u, x\left(g_{1}(u)\right), \ldots, x\left(g_{m}(u)\right)\right) \Delta u .
\end{aligned}
$$

Let $k \rightarrow \infty$. Then (3.7) gives

$$
x(t)=\left\{\begin{array}{l}
A+c(t) x(t-\tau)+\int_{t_{1}}^{t} \sigma(u) f\left(u, x\left(g_{1}(u)\right), \ldots, x\left(g_{m}(u)\right)\right) \Delta u+ \\
+\int_{t}^{\infty} t f\left(u, x\left(g_{1}(u)\right), \ldots, x\left(g_{m}(u)\right)\right) \Delta u, \quad \text { if } t \geq t_{1}, \\
x\left(t_{1}\right), \quad \text { if } \quad t_{0} \leq t<t_{1} .
\end{array}\right.
$$

Clearly, $x(t)>0$ on $\left[t_{0}, \infty\right)$. Therefore, $x(t)$ is a positive solution of (1.1). Since $0<A \leq x(t) \leq$ $\leq b_{1}$, from Theorem 3.1, $x(t) \in E(b, a, 0)$.

Theorem 3.3. Assume that $\lim _{t \rightarrow \infty} c(t)=c \in[0,1)$. Then Eq.(1.1) has a nonoscillatory solution $x(t) \in E(\infty, \infty, d)(d \neq 0)$ if and only if

$$
\int_{t_{0}}^{\infty}\left|f\left(u, h g_{1}(u), \ldots, h g_{m}(u)\right)\right| \Delta u<\infty \quad \text { for some } \quad h \neq 0
$$

Proof. Necessity. Without loss of generality, let $x(t) \in E(\infty, \infty, d)$ be an eventually positive solution of (1.1). From Theorem 3.1, we have that $d>0$. From (1.1) and (1.2) we have

$$
y^{\Delta \Delta}(t)+f\left(t, x\left(g_{1}(t)\right), \ldots, x\left(g_{m}(t)\right)\right)=0 .
$$


CLASSIFICATION AND EXISTENCE OF NONOSCILLATORY SOLUTIONS OF SECOND-ORDER NEUTRAL DELAY ... 199

Integrating it from $t_{1}$ to $t$, we get

$$
y^{\Delta}(t)-y^{\Delta}\left(t_{1}\right)+\int_{t_{1}}^{t} f\left(u, x\left(g_{1}(u)\right), \ldots, x\left(g_{m}(u)\right)\right) \Delta u=0 .
$$

Since $\lim _{t \rightarrow \infty} y^{\Delta}(t)=d>0$, we obtain

$$
\int_{t_{1}}^{\infty} f\left(u, x\left(g_{1}(u)\right), \ldots, x\left(g_{m}(u)\right)\right) \Delta u<\infty
$$

and there exist $d_{1}>0$ and $t_{2} \geq t_{1}$ such that $y(t) \geq d_{1} t$ for $t \geq t_{2}$. Therefore,

$$
\begin{aligned}
\int_{t_{1}}^{\infty} f\left(u, x\left(g_{1}(u)\right), \ldots, x\left(g_{m}(u)\right)\right) \Delta u & \geq \int_{t_{1}}^{\infty} f\left(u, y\left(g_{1}(u)\right), \ldots, y\left(g_{m}(u)\right)\right) \Delta u \geq \\
& \geq \int_{t_{1}}^{\infty} f\left(u, d_{1} g_{1}(u), \ldots, d_{1} g_{m}(u)\right) \Delta u .
\end{aligned}
$$

Choosing $h=d_{1}$ and combining (3.9) and (3.10), we get

$$
\int_{t_{1}}^{\infty} f\left(u, h g_{1}(u), \ldots, h g_{m}(u)\right) \Delta u<\infty .
$$

Sufficiency. Set $h>0$. Let $d>0, B>0$. From (3.8) there exists a sufficiently large $t_{1}$ so that for $t \geq t_{1}$ we have $t-\tau \geq t_{0}$ and $g_{i}(t) \geq t_{0}, i=1,2, \ldots, m$, and

$$
\frac{d}{h}+\frac{B}{t h}+c(t)+\frac{1}{h} \int_{t_{1}}^{\infty} f\left(u, h g_{1}(u), \ldots, h g_{m}(u)\right) \Delta u<1
$$

Define a set $\Omega$ by

$$
\Omega=\{z(t) \in X \mid d \leq z(t) \leq h\}
$$

and a operator $S$ on $\Omega$ by

$$
(S z)(t)=\left\{\begin{array}{l}
d+\frac{B}{t}+c(t) \frac{t-\tau}{t} z(t-\tau)+ \\
+\frac{1}{t} \int_{t_{1}}^{t} \sigma(u) f\left(u, g_{1}(u) z\left(g_{1}(u)\right), \ldots, g_{m}(u) z\left(g_{m}(u)\right)\right) \Delta u+ \\
+\int_{t}^{\infty} f\left(u, g_{1}(u) z\left(g_{1}(u)\right), \ldots, g_{m}(u) z\left(g_{m}(u)\right)\right) \Delta u, \quad \text { if } \quad t \geq t_{1}, \\
(S z)(t), \quad \text { if } \quad t_{0} \leq t<t_{1} .
\end{array}\right.
$$


Clearly, for $z(t) \in \Omega$

$$
\begin{aligned}
(S z)(t) \leq & d+\frac{B}{t}+c(t) h+\frac{1}{t} \int_{t_{1}}^{t} \sigma(u) f\left(u, h g_{1}(u), \ldots, h g_{m}(u)\right) \Delta u+ \\
& +\int_{t}^{\infty} f\left(u, h g_{1}(u), \ldots, h g_{m}(u)\right) \Delta u \leq d+\frac{B}{t}+c(t) h+ \\
& +\int_{t_{1}}^{\infty} f\left(u, h g_{1}(u), \ldots, h g_{m}(u)\right) \Delta u<h, \quad t \geq t_{1},
\end{aligned}
$$

and

$$
(S z)(t)=(S z)\left(t_{1}\right) \leq h, \quad t_{0} \leq t<t_{1} .
$$

It is easy to see that $(S z)(t) \geq d$ for $t \geq t_{0}$. Hence, $T \Omega \subset \Omega$. Define a series of sequences $\left\{z_{k}(t)\right\}, k \in \mathbf{N}$, by

$$
z_{0}(t)=d, \quad z_{k}(t)=\left(S z_{k-1}\right)(t), \quad t \geq t_{0}, \quad k \in \mathbf{N}_{\mathbf{0}} .
$$

We can prove that

$$
d \leq z_{k}(t) \leq z_{k+1}(t) \leq h, \quad t \geq t_{0}, \quad k \in \mathbf{N}_{\mathbf{0}}
$$

Then there exists $z(t) \in \Omega$ such that $\lim _{k \rightarrow \infty} z_{k}(t)=z(t), t \geq t_{0}$ and $d \leq z(t) \leq h$. Clearly, $z(t)=(S z)(t)\left(t \geq t_{0}\right)$, i.e.,

$$
z(t)=\left\{\begin{array}{l}
d+\frac{B}{t}+c(t) \frac{t-\tau}{t} z(t-\tau)+ \\
+\frac{1}{t} \int_{t_{1}}^{t} \sigma(u) f\left(u, g_{1}(u) z\left(g_{1}(u)\right), \ldots, g_{m}(u) z\left(g_{m}(u)\right)\right) \Delta u+ \\
+\int_{t}^{\infty} f\left(u, g_{1}(u) z\left(g_{1}(u)\right), \ldots, g_{m}(u) z\left(g_{m}(u)\right)\right) \Delta u, \quad \text { if } \quad t \geq t_{1}, \\
z\left(t_{1}\right), \quad \text { if } \quad t_{0} \leq t<t_{1} .
\end{array}\right.
$$

Let $x(t)=t z(t), t \geq t_{0}$. Then we have

$$
x(t)=\left\{\begin{array}{l}
d t+B+c(t) x(t-\tau)+ \\
+\int_{t_{1}}^{t} \sigma(u) f\left(u, x\left(g_{1}(u)\right), \ldots, x\left(g_{m}(u)\right)\right) \Delta u+ \\
+\int_{t}^{\infty} t f\left(u, x\left(g_{1}(u)\right), \ldots, x\left(g_{m}(u)\right)\right) \Delta u, \quad \text { if } t \geq t_{1}, \\
x\left(t_{1}\right), \quad \text { if } \quad t_{0} \leq t<t_{1} .
\end{array}\right.
$$


CLASSIFICATION AND EXISTENCE OF NONOSCILLATORY SOLUTIONS OF SECOND-ORDER NEUTRAL DELAY ...201

Hence, $x(t)$ is a positive solution of (1.1). On the other hand, $x(t) \geq y(t) \geq d t+B$. Hence $\lim _{t \rightarrow \infty} x(t)=\infty$ and $\lim _{t \rightarrow \infty} y(t)=\infty$. From (3.13), we have

$$
\begin{aligned}
y^{\Delta}(t) & =d+\int_{t}^{\infty} f\left(u, x\left(g_{1}(u)\right), \ldots, x\left(g_{m}(u)\right)\right) \Delta u= \\
& =d+\int_{t}^{\infty} f\left(u, g_{1}(u) z\left(g_{1}(u)\right), \ldots, g_{m}(u) z\left(g_{m}(u)\right)\right) \Delta u \leq \\
& \leq d+\int_{t}^{\infty} f\left(u, h g_{1}(u), \ldots, h g_{m}(u)\right) \Delta u .
\end{aligned}
$$

Hence, $\lim _{t \rightarrow \infty} y^{\Delta}(t)=d$. Therefore, $x(t) \in E(\infty, \infty, d)$.

Theorem 3.4. Assume that $\lim _{t \rightarrow \infty} c(t)=c \in[0,1)$. Further, assume that

$$
\int_{t_{0}}^{\infty}\left|f\left(u, h g_{1}(u), \ldots, h g_{m}(u)\right)\right| \Delta u<\infty \quad \text { for some } \quad h \neq 0
$$

and

$$
\int_{t_{0}}^{\infty} \sigma(u)\left|f\left(u, b_{1}, \ldots, b_{1}\right)\right| \Delta u=\infty \quad \text { for some } \quad b_{1} \neq 0,
$$

where $b_{1} h>0$. Then Eq. (1.1) has a nonoscillatory solution $x(t) \in E(\infty, \infty, 0)$.

Proof. Without loss of generality, assume that $h>0$ and $b_{1}>0$. From (3.14) there exists a sufficiently large $t_{1}$ so that for $t \geq t_{1}$ we have $t-\tau \geq t_{0}$ and $g_{i}(\sigma(t)) \geq t_{0}, i=1,2, \ldots, m$, and

$$
\frac{b_{1}}{t h}+c(t)+\frac{1}{h} \int_{t_{1}}^{\infty} f\left(u, h g_{1}(u), \ldots, h g_{m}(u)\right) \Delta u<1 .
$$

Define a set $\Omega$ by

$$
\Omega=\left\{z(t) \in X \mid 0 \leq z(t) \leq h, t \geq t_{0}\right\}
$$

and an operator $S$ on $\Omega$ by

$$
(S z)(t)=\left\{\begin{array}{l}
\frac{b_{1}}{t}+c(t) \frac{t-\tau}{t} z(t-\tau)+ \\
+\frac{1}{t} \int_{t_{1}}^{t} \sigma(u) f\left(u, g_{1}(u) z\left(g_{1}(u)\right), \ldots, g_{m}(u) z\left(g_{m}(u)\right)\right) \Delta u+ \\
+\int_{t}^{\infty} f\left(u, g_{1}(u) z\left(g_{1}(u)\right), \ldots, g_{m}(u) z\left(g_{m}(u)\right)\right) \Delta u, \quad \text { if } \quad t \geq t_{1}, \\
(S z)(t), \quad \text { if } \quad t_{0} \leq t<t_{1} .
\end{array}\right.
$$

ISSN 1562-3076. Нелінійні коливання, 2013, m. 16, № 2 
Clearly, for $z(t) \in \Omega$,

$$
\begin{aligned}
(S z)(t) \leq & \frac{b_{1}}{t}+c(t) h+\frac{1}{t} \int_{t_{1}}^{t} \sigma(u) f\left(u, h g_{1}(u), \ldots, h g_{m}(u)\right) \Delta u+ \\
& +\int_{t}^{\infty} f\left(u, h g_{1}(u), \ldots, h g_{m}(u)\right) \Delta u \leq \frac{b_{1}}{t}+c(t) h+ \\
& +\int_{t_{1}}^{\infty} f\left(u, h g_{1}(u), \ldots, h g_{m}(u)\right) \Delta u \leq h, \quad t \geq t_{1},
\end{aligned}
$$

and $(S z)(t)=(S z)\left(t_{1}\right) \leq h, t_{0} \leq t<t_{1}$, i.e., $S \Omega \subset \Omega$.

Define a series of sequences $\left\{z_{k}(t)\right\}, k \in \mathbf{N}$, by

$$
z_{0}(t)=0, \quad z_{k}(t)=\left(S z_{k-1}\right)(t), \quad t \geq t_{0}, \quad k \in \mathbf{N}_{\mathbf{0}} .
$$

By induction, we can prove that

$$
0 \leq z_{k}(t) \leq z_{k+1}(t) \leq h, \quad t \geq t_{0}, \quad k \in \mathbf{N} .
$$

Then there exists $z(t) \in \Omega$ such that $\lim _{k \rightarrow \infty} z_{k}(t)=z(t), t \geq t_{0}$.

Clearly, $z(t)=(S z)(t), t \geq t_{0}$, i.e.,

$$
z(t)=\left\{\begin{array}{l}
\frac{b_{1}}{t}+c(t) \frac{t-\tau}{t} z(t-\tau)+ \\
+\frac{1}{t} \int_{t_{1}}^{t} \sigma(u) f\left(u, g_{1}(u) z\left(g_{1}(u)\right), \ldots, g_{m}(u) z\left(g_{m}(u)\right)\right) \Delta u+ \\
+\int_{t}^{\infty} f\left(u, g_{1}(u) z\left(g_{1}(u)\right), \ldots, g_{m}(u) z\left(g_{m}(u)\right)\right) \Delta u, \quad \text { if } \quad t \geq t_{1}, \\
z\left(t_{1}\right), \quad \text { if } \quad t_{0} \leq t<t_{1} .
\end{array}\right.
$$

Let $x(t)=t z(t), t \geq t_{0}$. Then we have

$$
x(t)=\left\{\begin{array}{l}
b_{1}+c(t) x(t-\tau)+ \\
+\int_{t_{1}}^{t} \sigma(u) f\left(u, x\left(g_{1}(u)\right), \ldots, x\left(g_{m}(u)\right)\right) \Delta u+ \\
+\int_{t}^{\infty} t f\left(u, x\left(g_{1}(u)\right), \ldots, x\left(g_{m}(u)\right)\right) \Delta u, \text { if } t \geq t_{1}, \\
x\left(t_{1}\right), \quad \text { if } t_{0} \leq t<t_{1} .
\end{array}\right.
$$

Hence, $x(t)$ is a positive solution of (1.1). On the other hand, from (3.18), we have $x(t) \geq b_{1}$ and that

$$
x(t) \geq y(t)=x(t)-c(t) x(t-\tau) \geq \int_{t_{1}}^{t} \sigma(u) f\left(u, b_{1}, \ldots, b_{1}\right) \Delta u
$$


CLASSIFICATION AND EXISTENCE OF NONOSCILLATORY SOLUTIONS OF SECOND-ORDER NEUTRAL DELAY... 203

which together with (3.15) imply $\lim _{t \rightarrow \infty} x(t)=\infty$ and $\lim _{t \rightarrow \infty} y(t)=\infty$. By (3.18), we get

$$
\begin{aligned}
y^{\Delta}(t) & =\int_{t}^{\infty} f\left(u, x\left(g_{1}(u)\right), \ldots, x\left(g_{m}(u)\right)\right) \Delta u= \\
& =\int_{t}^{\infty} f\left(u, g_{1}(u) z\left(g_{1}(u)\right), \ldots, g_{m}(u) z\left(g_{m}(u)\right)\right) \Delta u \leq \\
& \leq \int_{t}^{\infty} f\left(u, h g_{1}(u), \ldots, h g_{m}(u)\right) \Delta u .
\end{aligned}
$$

Hence

$$
0 \leq \lim _{t \rightarrow \infty} y^{\Delta}(t) \leq \lim _{t \rightarrow \infty} \int_{t}^{\infty} f\left(u, h g_{1}(u), \ldots, h g_{m}(u)\right) \Delta u=0
$$

i.e., $\lim _{t \rightarrow \infty} y^{\Delta}(t)=0$. Therefore, $x(t) \in E(\infty, \infty, 0)$.

Theorem 3.5. Assume that $\lim _{t \rightarrow \infty} c(t)=c \in[0,1)$. Further assume that there exists $d>0$ such that

$$
\int_{t_{0}}^{\infty} f\left(u, d_{1}, \ldots, d_{1}\right) \Delta u=\infty \quad \text { for any } \quad d_{1} \in(0, d]
$$

Then every solution $x(t)$ of Eq. (1.1) either oscillates or $\{x(t)\} \in E(0,0,0)$.

Proof. Let $x(t)$ be an eventually positive solution of (1.1). By Lemma 3.1, if $\lim _{t \rightarrow \infty} x(t)=$ $=0$, then $\lim _{t \rightarrow \infty} y(t)=0$ and so $\lim _{t \rightarrow \infty} y^{\Delta}(t)=0$. Hence, $x(t) \in E(0,0,0)$. If $\lim _{t \rightarrow \infty} x(t)=$ $=0$ fails, then $y(t)>0$ eventually. Since $y^{\Delta \Delta}(t)<0$, we have $y^{\Delta}(t)>0$, eventually. Therefore, there exists $\bar{d} \in(0, d]$ such that $x(t) \geq y(t) \geq \bar{d}$. From (1.1) and (1.2), we have

$$
y^{\Delta \Delta}(t)=-f\left(t, x\left(g_{1}(t)\right), \ldots, x\left(g_{m}(t)\right)\right) .
$$

Integrating it from $t_{0}$ to $t$, we obtain

$$
y^{\Delta}(t)-y^{\Delta}\left(t_{0}\right)=-\int_{t_{0}}^{t} f\left(u, x\left(g_{1}(u)\right), \ldots, x\left(g_{m}(u)\right)\right) \Delta u \leq-\int_{t_{0}}^{t} f(u, \bar{d}, \ldots, \bar{d}) \Delta u
$$

Let $t \rightarrow \infty$. Then we get $\int_{t_{0}}^{\infty} f(u, \bar{d}, \ldots, \bar{d}) \Delta u<\infty$ which contradicts (3.19) and completes the proof. 
The above results can be extended to the second order neutral equation

$$
[x(t)-c(t) x(t-\tau)]^{\Delta \Delta}=f\left(t, x\left(g_{1}(t)\right), \ldots, x\left(g_{m}(t)\right)\right)
$$

With respect to equation (3.20), we assume that conditions $\left(\mathrm{H}_{1}\right),\left(\mathrm{H}_{2}\right)$ and $\left(\mathrm{H}_{3}\right)$ hold. By the same argument, we have the following theorems.

Theorem 3.6. Assume that $\lim _{t \rightarrow \infty} c(t)=c \in[0,1)$. Let $x(t)$ be a nonoscillatory solution of (3.20). Let $S$ denote the set of all nonoscillatory solution of (3.20), and define

$$
\begin{gathered}
E(0,0,0)=\left\{x(t) \in E: \lim _{t \rightarrow \infty} x(t)=0, \lim _{t \rightarrow \infty} y(t)=0, \lim _{t \rightarrow \infty} y^{\Delta}(t)=0\right\} \\
E(b, a, 0)=\left\{x(t) \in E: \lim _{t \rightarrow \infty} x(t)=b=\frac{a}{1-c}, \lim _{t \rightarrow \infty} y(t)=a, \lim _{t \rightarrow \infty} y^{\Delta}(t)=0\right\} \\
E(\infty, \infty, d)=\left\{x(t) \in E: \lim _{t \rightarrow \infty} x(t)=\infty, \lim _{t \rightarrow \infty} y(t)=\infty, \lim _{t \rightarrow \infty} y^{\Delta}(t)=d \neq 0\right\} \\
E(\infty, \infty, \infty)=\left\{x(t) \in E: \lim _{t \rightarrow \infty} x(t)=\infty, \lim _{t \rightarrow \infty} y(t)=\infty, \lim _{t \rightarrow \infty} y^{\Delta}(t)=\infty\right\}
\end{gathered}
$$

Then

$$
E=E(0,0,0) \cup E(b, a, 0) \cup E(\infty, \infty, d) \cup E(\infty, \infty, \infty)
$$

Theorem 3.7. Assume that $\lim _{t \rightarrow \infty} c(t)=c \in[0,1)$. Further, assume that

$$
\int_{t_{0}}^{\infty} \sigma(u)\left|f\left(u, b_{1}, \ldots, b_{1}\right)\right| \Delta u<\infty \quad \text { for some } \quad b_{1} \neq 0 .
$$

Then Eq. (3.20) has a nonoscillatory solution $x(t) \in E(b, a, 0)(b \neq 0, a \neq 0)$.

Theorem 3.8. Assume that $\lim _{t \rightarrow \infty} c(t)=c \in[0,1)$. Then the following statements are true.

(i) If Eq. (3.20) has a nonoscillatory solution $x(t) \in E(\infty, \infty, d), d \neq 0$, then

$$
\int_{t_{0}}^{\infty}\left|f\left(u, h g_{1}(u), \ldots, h g_{m}(u)\right)\right| \Delta u<\infty, \quad \text { for some } h \neq 0 .
$$

(ii) If

$$
\int_{t_{0}}^{\infty} \sigma(u)\left|f\left(u, h g_{1}(u), \ldots, h g_{m}(u)\right)\right| \Delta u<\infty, \quad \text { for some } h \neq 0,
$$

then Eq. (3.20) has a nonoscillatory solution $x(t) \in E(\infty, \infty, d), d \neq 0$.

Theorem 3.9. Assume that $\lim _{t \rightarrow \infty} c(t)=c \in[0,1)$. Further assume that there exists $d>0$ such that

$$
\int_{t_{0}}^{\infty} f\left(u, d_{1}, \ldots, d_{1}\right) \Delta u=\infty \quad \text { for any } \quad d_{1} \in(0, d] .
$$


CLASSIFICATION AND EXISTENCE OF NONOSCILLATORY SOLUTIONS OF SECOND-ORDER NEUTRAL DELAY... 205

Then every solution $x(t)$ of $E q$. (1.1) either oscillates or $x(t) \in E(0,0,0)$, or $x(t) \in E(\infty, \infty, \infty)$.

In the following, we shall give some examples.

Example 3.1. In the case where $T=\mathbf{R}$, we consider the second order differential equation

$$
\left(x(t)-\frac{1}{2} x(t-\tau)\right)^{\prime \prime}+\frac{2(t-1)^{3}-t^{3}}{(t-1)^{6}} x^{3}(t)=0 .
$$

(3.4) becomes

$$
\int_{t_{0}}^{\infty} u\left|f\left(u, b_{1}, \ldots, b_{1}\right)\right| d u<\infty \text { for some } \quad b_{1} \neq 0 .
$$

It is easy to see that (3.22) holds. Therefore, (3.21) has a nonoscillatory solution $x(t) \in E(b, a, 0)$, $b \neq 0, a \neq 0$. In fact, $x(t)=1-\frac{1}{t}$ is such a solution, where $a=\frac{1}{2}, b=1$.

Example 3.2. In the case where $T=\mathbf{N}$, we consider the second order delay difference equation

$$
\Delta^{2}\left(x_{n}-\frac{1}{4} x_{n-1}\right)+\frac{2^{-n-3}}{\left(n-1-2^{-n+1}\right)^{5}} x_{n-1}^{5}=0, \quad n \geq 2,
$$

for which (3.8) becomes

$$
\sum_{j=n_{0}}^{\infty}\left|f\left(j, h g_{1}(j), \ldots, h g_{m}(j)\right)\right|<\infty
$$

It is easy to see that (3.24) is satisfied. In fact, the sequence $x_{n}=\left\{n-\frac{1}{2^{n}}\right\}$ is a nonoscillatory solution of (3.23) which belongs to the class $S\left(\infty, \infty, \frac{3}{4}\right)$.

Example 3.3. In the case where $T=h \mathbf{Z}=\{h k \mid k \in \mathbf{Z}\}$ for $h>1$, we consider the second order delay dynamic equation

$$
\left[x(t)-\frac{1}{2} x(t-h)\right]^{\Delta \Delta}+\frac{\left(\frac{1}{2}-2^{-h}\right)\left(2^{-h}-1\right)^{2}}{h^{2}} x(t-h)=0
$$

for which condition (3.19) of Theorem 3.5 is satisfied. In fact, $x(t)=\frac{1}{2^{t}}$ is a nonoscillatory solution of (3.25) which belongs to the class $S(0,0,0)$.

1. Agarwal R. P., Bohner M. Basic calculus on time scales and some of its applications // Results Math. 1999. - 35. - P. 3-22.

2. Agarwal R. P., Bohner M., O’Regan D., Peterson A. Dynamic equations on time scales: a survey // J. Comput. Appl. Math. - 2002. - 141. - P. 1-26. 
3. Agarwal R. P., O’Regan D., Saker S. H. Oscillation creteria for second-order nonlinear neutral delay dynamic equations // J. Math. Anal. and Appl. - 2004. - 300. - P. 203-217.

4. Bohner M., Peterson A. Dynamic equations on time scales: An Introduction with Applications. - Boston: Birkhäuser, 2001.

5. Bohner M., Peterson A. Advance in dynamic equations on time scales. - Boston: Birkhäuser, 2003.

6. Bohner M., Erbe L., Peterson A. Oscillation for nonlinear second order dynamic equations on a time scale // J. Math. Anal. and Appl. - 2005. - 301. - P. $491-507$.

7. Bohner M., Saker S. H. Oscillation criteria for perturbed nonlinear dynamic equations // Math. Comput. Modelling. - 2004. - 40. - P. 249-260.

8. Erbe L., Peterson A. Oscillation criteria for second-order matrix dynamic equations on a time scale // J. Comput. Appl. Math. - 2002. - 141. - P. 169-185.

9. Erbe L., Peterson A., Saker S. H. Asymptotic behavior of solutions of a third-order nonlinear dynamic equation on time scales // J. Comput. Appl. Math. - 2005. - 181. - P. $92-102$.

10. Hilger S. Ein Maßkettenkalkül mit Anwendung auf Zentrumsmannigfaltigkeiten: Ph.D. thesis. - Univ. Würzburg, 1988.

11. Kaymakcalan B., Lakshmikantham V., Sivasundaram S. Dynamic systems on measure chains. - Dordrecht: Kluwer Acad., 1996.

12. Medico A. D., Kong $Q$. $K$ Kamenev-type and interval oscillation criteria for second-order linear differential equations on a measure chain // J. Math. Anal. and Appl. - 2004. - 294. - P. 621-643.

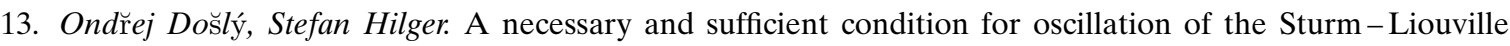
dynamic equation on time scales // J. Comput. Appl. Math. - 2002. - 141, № 1-2. - P. 147-158.

14. Saker S. H. Oscillation criteria of second-order half-linear dynamic equations on time scales // J. Comput. Appl. Math. - 2005. - 177. - P. 375-387.

15. Saker S. H. Oscillation of nonlinear dynamic equations on time scales // Appl. Math. Comput. - 2004. 148. - P. 81-91.

16. Saker S. H. Oscillation of second-order nonlinear neutral delay dynamic equations on time scales // J. Comput. Appl. Math. - 2005.

17. Zhang B. G., Deng X. H. Oscillation of delay differential equations on time scales // Math. Comput. Modelling. - 2002. - 36. - P. $1307-1318$.

18. Zhang B. G., Zhu S. L. Oscillation of second-order nonlinear delay dynamic equations on time scales // Comput. Math. Appl. - 2005. - 49. - P. 599-609.

19. Yong Zhou, Zhang B. G. Classification and existence of nonoscillatory solutions of second-order neutral delay difference equations // Z. Anal. und Anwendungen. - 2001. - 20. - S. 223-234.

20. Yong Zhou. Oscillation and non-oscillation criteria for second order quasilinear difference equations // J. Math. Anal. and Appl. - 2005. - 303. - P. 365-375. 